Corrigendum

\title{
Corrigendum to "Molecular Characterization of a Fully Human Chimeric T-Cell Antigen Receptor for Tumor-Associated Antigen EpCAM"
}

\author{
Naoto Shirasu, ${ }^{1,2}$ Hiromi Yamada, ${ }^{1}$ Hirotomo Shibaguchi, ${ }^{1,2}$ \\ Motomu Kuroki, ${ }^{3}$ and Masahide Kuroki ${ }^{1,2}$ \\ ${ }^{1}$ Department of Biochemistry, Faculty of Medicine, Fukuoka University, 7-45-1 Nanakuma, Jonan-ku, Fukuoka 814-0180, Japan \\ ${ }^{2}$ Center for Advanced Molecular Medicine, Fukuoka University, 7-45-1 Nanakuma, Jonan-ku, Fukuoka 814-0180, Japan \\ ${ }^{3}$ School of Nursing, Faculty of Medicine, Fukuoka University, 7-45-1 Nanakuma, Jonan-ku, Fukuoka 814-0180, Japan \\ Correspondence should be addressed to Naoto Shirasu; shirasu@fukuoka-u.ac.jp
}

Received 25 November 2014; Accepted 30 November 2014

Copyright (c) 2015 Naoto Shirasu et al. This is an open access article distributed under the Creative Commons Attribution License, which permits unrestricted use, distribution, and reproduction in any medium, provided the original work is properly cited.

In the paper entitled "Molecular Characterization of a Fully Human Chimeric T-Cell Antigen Receptor for Tumor-Associated Antigen EpCAM," there was an error in the amino acid/ nucleotide sequences in Figure 2. Actually, the sequences were not for anti-EpCAM scFv but for anti-CEA scFv, which has been cloned by our laboratory in the previous study. Here we provide the correct version of Figure 2. 


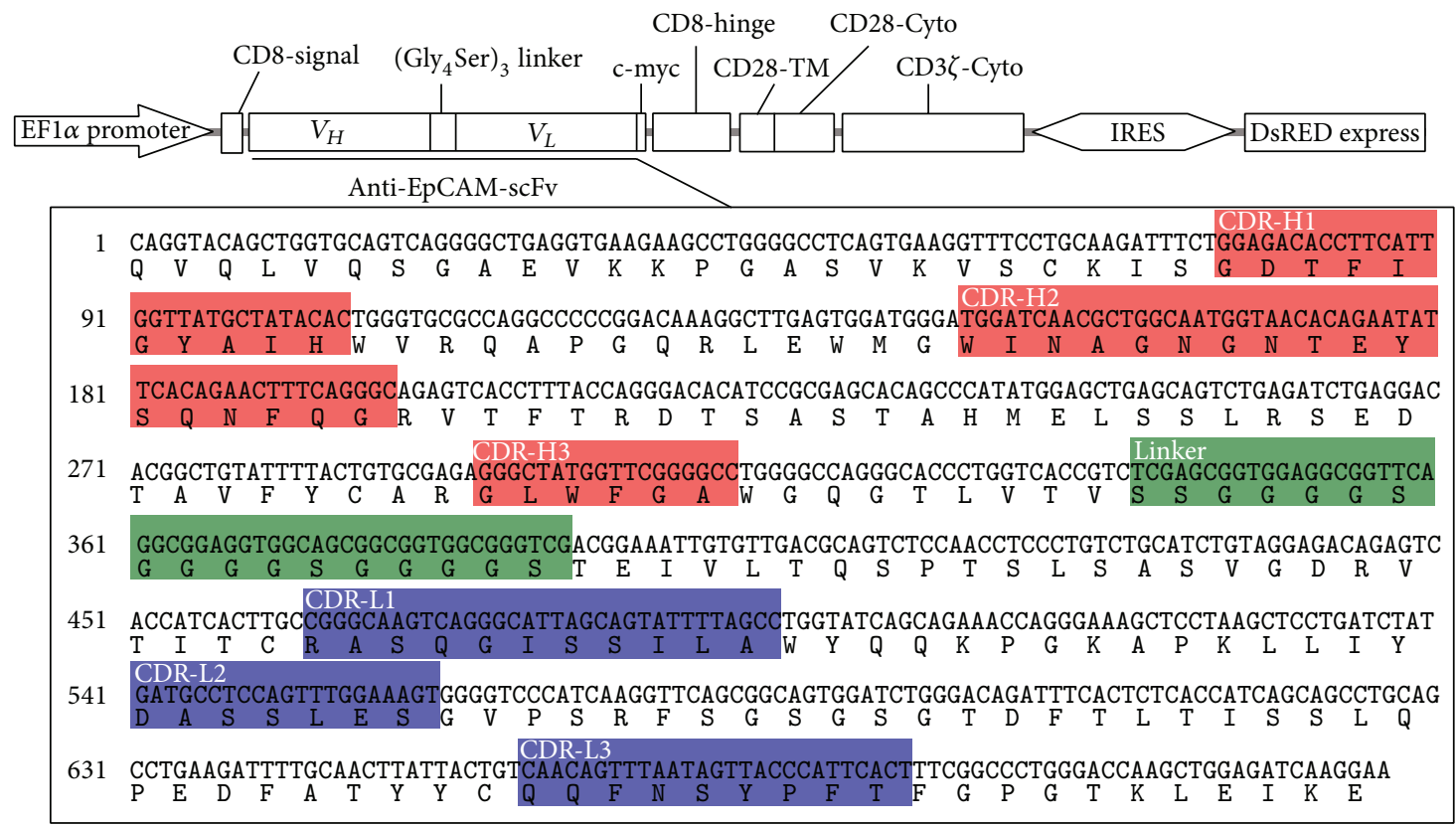

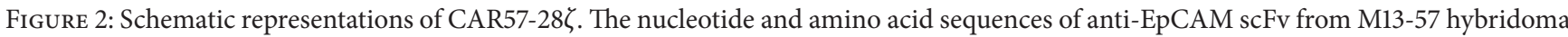
were analyzed using the IMGT/V-QUEST and IMGT/JunctionAnalysis online tools (http://www.imgt.org/), and each complementarity determining region (CDR) of the cloned scFv gene was determined according to Chothia's definition [15]. VH, variable region of heavy chain; VL, variable region of light chain; TM, transmembrane domain; Cyto, cytoplasmic domain. 

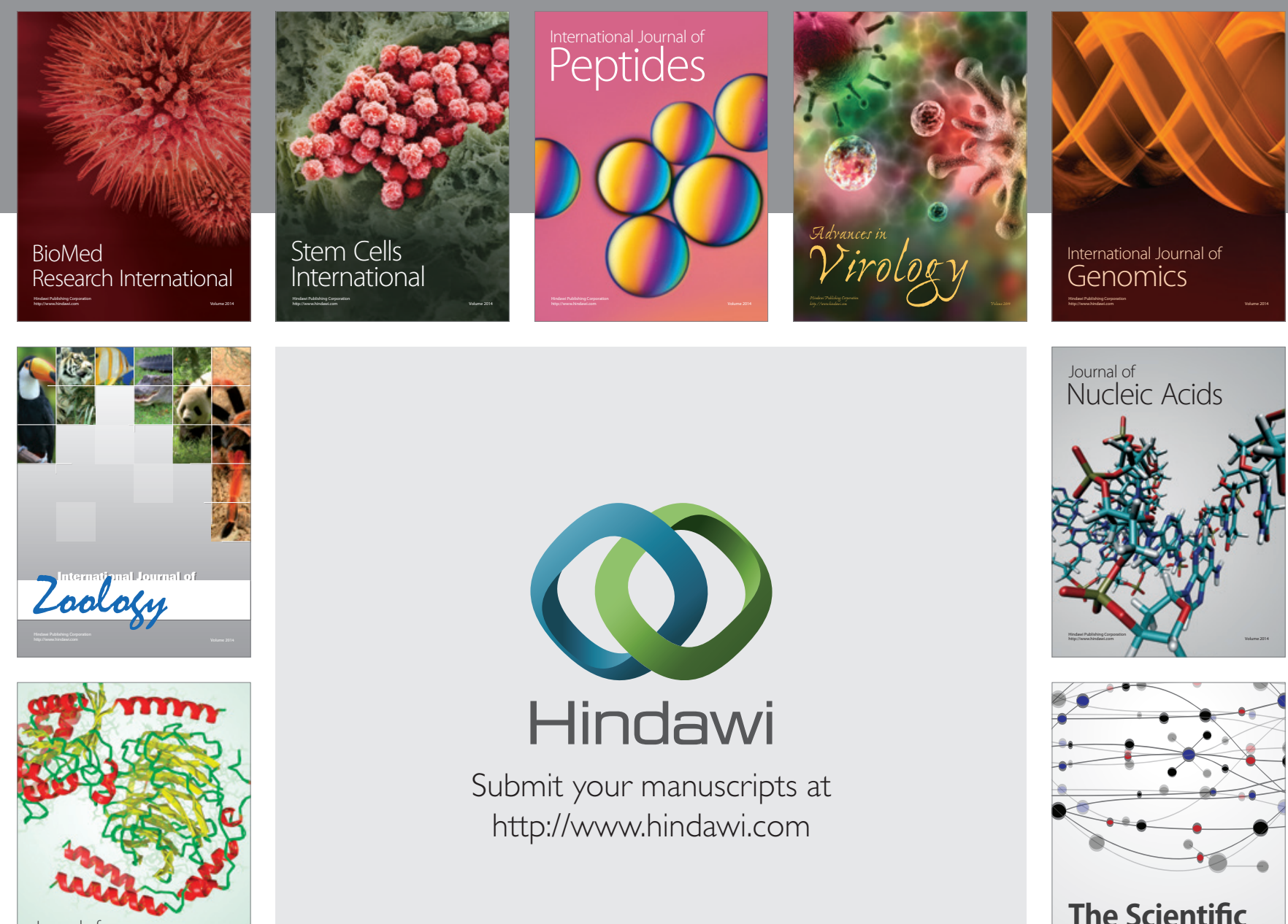

Submit your manuscripts at

http://www.hindawi.com

Journal of
Signal Transduction
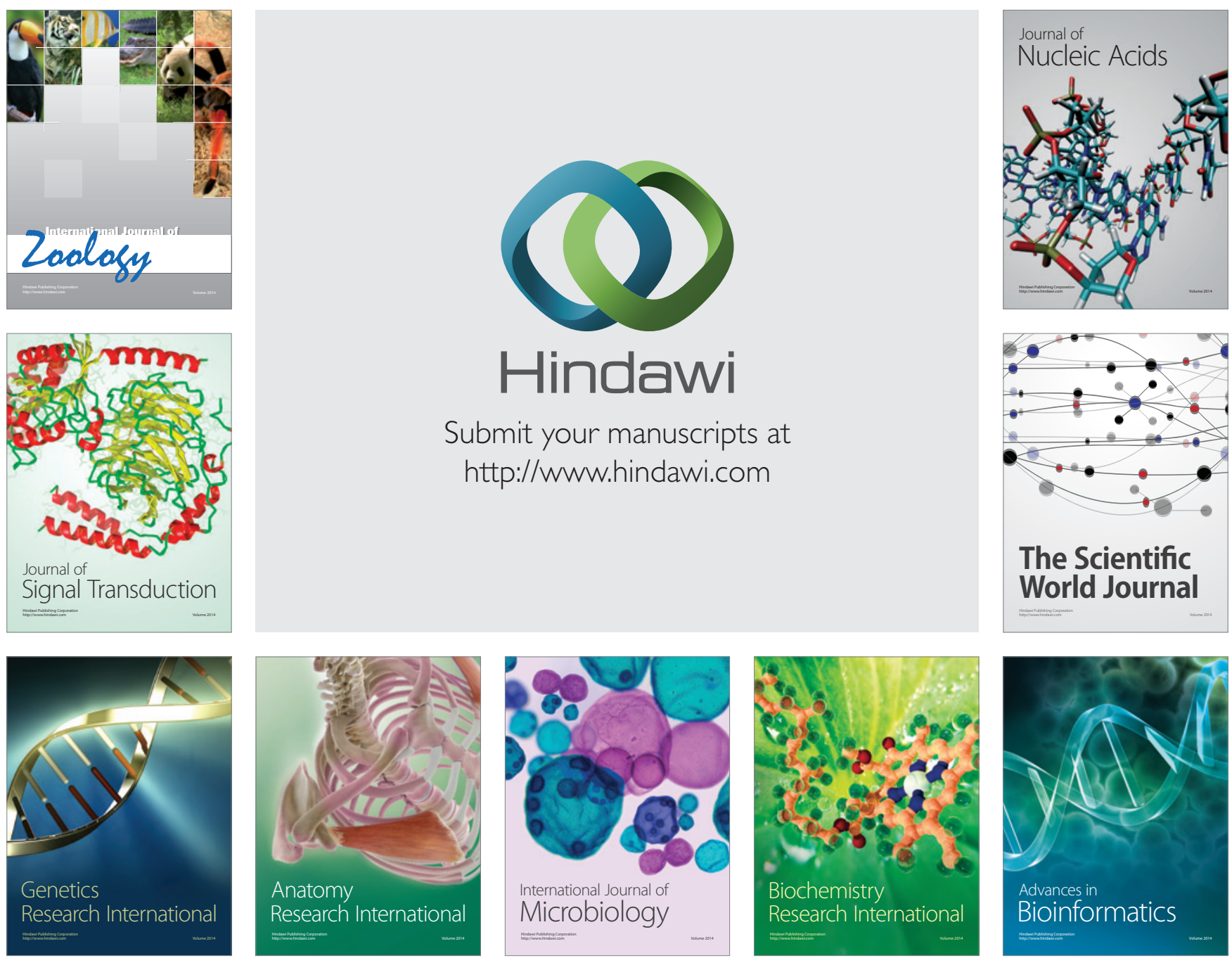

The Scientific World Journal
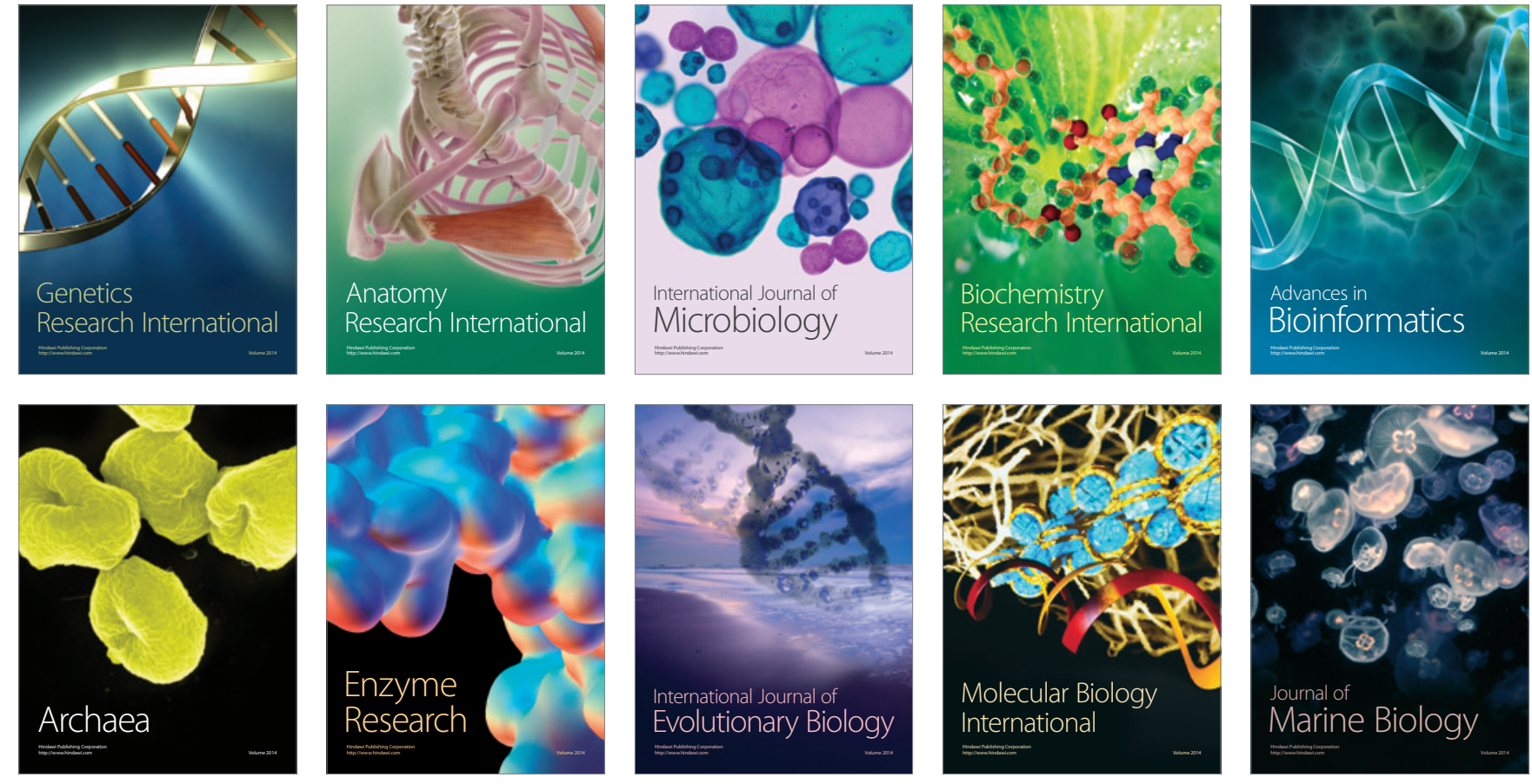